\title{
Molecular characterisation of glutamate dehydrogenase gene defects in Japanese patients with congenital hyperinsulinism/hyperammonaemia
}

\author{
Hiroki Fujioka $^{1}$, Yoshiyuki Okano*, ${ }^{*}$, Hiroshi Inada ${ }^{1}$, Minoru Asada $^{1}$, Tomoyuki Kawamura ${ }^{1}$ \\ Yutaka Hase $^{2}$ and Tsunekazu Yamano ${ }^{1}$ \\ ${ }^{1}$ Department of Pediatrics, Osaka City University Graduate School of Medicine, Osaka, Japan; ${ }^{2}$ Osaka City \\ Environment and Public Health Center, Osaka, Japan
}

Congenital hyperinsulinism and hyperammonaemia $(\mathrm{CHH})$ is caused by dysregulation of glutamate dehydrogenase (GDH). We characterised the GDH gene in two Japanese patients with $\mathrm{CHH}$. Patient 1 showed late-onset and mild hypoglycaemic episodes and mild hyperammonaemia, compared with patient 2. In GDH activity of lymphoblasts, patient 1 showed twofold higher basal GDH activity than control subjects and mild insensitivity for GTP inhibition. Patient 2 showed severe insensitivity for GTP inhibition, and similar allosteric stimulation by ADP in the controls. Genetic studies identified heterozygous and de novo L413V and G446D mutations in patients 1 and 2, respectively. COS cell expression study confirmed that both mutations were disease-causing gene. The insensitivity for GTP inhibition in L413V and G446D was emphasised in COS cell expression system as a result of the dosage effect of mutant GDH gene. L413V showed less impairment of GDH than G446D based on biochemical and genetic results, which was consistent with the clinical phenotype. Based on the structure of bovine GDH, G446D was located in GTP binding site of pivot helix and its surroundings, while L413V was located in $\alpha$-helix of antenna-like structure. These different locations of mutations gave different effects on GDH enzyme. The antenna-like structure plays an important role in GDH activity.

European Journal of Human Genetics (2001) 9, 931 -937.

Keywords: glutamate dehydrogenase; hyperinsulinism; hyperammonaemia; hypoglycaemia

\section{Introduction}

Hypoglycaemia in neonatal and early infantile period can be due to a variety of causes. The majority of such cases have transient hypoglycaemia caused by perinatal suffering such as immaturity, asphyxia, diabetic mother, and infection. Persistent hypoglycaemia is caused by innate abnormality as hyperplasia of pancreatic $\beta$-cells and focal disease. Recent reports have indicated that the underlying pathology in

*Correspondence: Y Okano, Department of Pediatrics, Osaka City University Graduate School of Medicine, 1-4-3 Asahimachi, Abeno-ku, Osaka 545-8585, Japan. Tel: +81 66645 3816; Fax: +81 66636 8737; E-mail: okano@med.osaka-cu.ac.jp

Received 10 August 2001; revised 18 October 2001; accepted 22 October 2001 some patients with hyperinsulinism-related persistent hypoglycaemia includes genetic disorders that result in inhibition of the signal transduction pathways regulating the secretion of insulin, including disorders of glucokinase, ${ }^{1}$ sulphonylurea receptor $^{2,3}$ and inward rectifier potassium channels (Kir6.2) genes. $^{4,5}$

Congenital hyperinsulinism and hyperammonaemia (CHH, OMIM 138130) is a disorder characterised by symptomatic hypoglycaemia and persistently high plasma ammonium levels. ${ }^{6-8}$ In $\mathrm{CHH}$, diazoxide is most effective for hypoglycaemia but protein restriction and/or treatment with sodium benzoate are ineffective against hyperammonaemia. The pathogenesis of concurrent hyperinsulinism and hyperammonaemia in patients with $\mathrm{CHH}$ was smartly resolved by Stanley et $a l ;{ }^{9}$ dysregulation of glutamate dehydrogenase 
(GDH, EC 1.4.1.3) by missense mutations in GTP binding site of exons 11 and $12^{10,11}$ causes inappropriate secretion of insulin by pancreatic $\beta$-cells and affect nitrogen metabolism in liver.

GDH is a mitochondrial enzyme that catalyses reversible oxidative deamination of L-glutamate to 2-oxoglutarate using cofactors of $\mathrm{NAD}^{+}$and/or $\mathrm{NADP}^{+}$. GDH is controlled by allosteric effectors of inhibition by GTP and NADH and activation by $\mathrm{ADP}, \mathrm{NAD}^{+}$, and leucine. Patients with $\mathrm{CHH}$ show less sensitivity to inhibition by GTP for the altered form in the GTP binding site of GDH. It was proposed that this altered form of GDH leads to higher oxidation rates and ATP production. In pancreatic $\beta$-cells, any increase in the ATP/ ADP ratio results in closure of the $\mathrm{K}^{+}$channels and depolarisation of $\beta$-cells. This depolarisation causes calcium influx and the release of stored insulin granules. ${ }^{12}$ In the liver and other tissues, activated GDH could consume massive amounts of glutamate and produce ammonia. In addition to this, production of N-acetylglutamate, which is one of the positive effectors of carbamoyl phosphate synthetase, might decrease due to a reduction in the amount of glutamate in hepatocytes. ${ }^{13}$

In the present study, we report two patients with $\mathrm{CHH}$, in whom we identified dysregulation of GDH activity and missense mutation in a single allele. We also demonstrated that the two mutations in different locations generated dysregulation of GDH activity with different mechanism and that the clinical phenotypes correlated with the genotypes.

\section{Materials and methods Patients}

Patient 1, a male, was born at 40 weeks of gestation, weighing $3096 \mathrm{~g}$, by uneventful delivery. He was the first live-born child of unrelated parents with no family history of hypoglycaemia or hyperammonaemia. At 4 months of age, he developed recurrent tonic seizure with hypoglycaemia $(0.72 \mathrm{mmol} / \mathrm{l})$ and hyperammonaemia $(132 \mu \mathrm{mol} / \mathrm{l})$. At 7 months of age, he was admitted to a hospital and diagnosed with leucine-sensitive hypoglycaemia. The patient was treated with dietary control of low leucine and low protein, sodium benzoate, and $l$-carnitine. However, hypoglycaemic episodes and hyperammonaemia continued after such treatment and the patient was referred to our hospital at 7 years of age. WISC-R intellectual test showed an IQ of 70 and he was reported to suffer from somnolence and exhibited a desultory attitude at school. The patient was treated with 5 to $7 \mathrm{mg} / \mathrm{kg} /$ day of diazoxide to improve blood glucose levels, which resulted in a reduction of hypoglycaemic episodes. The IQ improved to 100 by the WISC-R intellectual test and he showed good attitude in school at 10 years of age. However, the levels of ammonia remained at 82 to $138 \mu \mathrm{mol} /$ $1(116 \pm 15.9 \mu \mathrm{mol} / \mathrm{l}$, mean $\pm \mathrm{SD}$ of 20 samples $)$ in the last 3 years.
Patient 2, a male, was born at 40 weeks of gestation, weighing $3275 \mathrm{~g}$, by uneventful delivery. He was the first live-born child of unrelated parents with no family history of hypoglycaemia or hyperammonaemia. At 5 days old, he developed hypothermia and became inactive. He was admitted to the hospital for severe hypoglycaemia $(0.44 \mathrm{mmol} / \mathrm{l})$ and hyperinsulinism and was treated with diazoxide. However, hypoglycaemia persisted, and leucine tolerance test showed prolonged and excessive insulin secretion. At 6 months of age, subtotal pancreatomy was performed for uncontrolled hypoglycaemia. Subsequently, the hepatomegaly and hyperammonaemia $(147 \mu \mathrm{mol} / \mathrm{l})$ was discovered. Liver biopsy showed severe fatty changes but biochemical analysis showed normal activities of urea cycle enzymes. Subtotal pancreatomy failed to reduce hypoglycaemic episodes and seizures. Furthermore, sodium benzoate and l-carnitine were ineffective in controlling hyperammonaemia. The patient was referred to our hospital at 11 months of age following a change of residential address. The score of Tsumori-Inage's developing test (DQ) was 57 at 2 years of age (normal score: more than 80). Hypoglycaemic episodes persisted but gradually decreased in frequency up to 8 years, though the patient was continuously treated with diazoxide. At 9 years, blood glucose level gradually increased over the normal range. Percutaneous insulin replacement therapy commenced for relative insulin insufficiency and not by the cause of insulin resistance related to obesity. The levels of ammonia remained at 116 to $176 \mu \mathrm{mol} / 1(142 \pm 17 \mu \mathrm{mol} / 1$, mean \pm SD of 31 samples) in the last 3 years. At the latest follow-up, the patient showed moderate level of mental retardation and abnormal behaviour such as hyperkinesia.

Informed consent for genetic analysis was obtained from all subjects or their parents.

\section{Measurement of GDH activity}

An Epstein-Barr virus (EBV)-transformed lymphoblastoid cell line was established from the patients and control subjects, and was used for enzymatic and molecular analyses. The lymphoblastoid cells of patients and controls were homogenised in $10 \mathrm{~mm}$ Tris-acetate ( $\mathrm{pH}$ 8.0) and $1 \mathrm{~mm}$ EDTA with a Potter-Elvehjem homogeniser on ice. The cell lysate was centrifuged at $350 \times g$ for $10 \mathrm{~min}$ at $4^{\circ} \mathrm{C}$, and the supernatant was used as the enzyme source. The enzyme source was preincubated with $20 \mathrm{~mm}$ Tris-acetate (pH 8.0), $50 \mathrm{~mm} \mathrm{NH}_{4} \mathrm{Cl}, 0.2 \mathrm{~mm} \mathrm{NADH}, 1 \mathrm{~mm}$ EDTA, and $0.1 \%$ Triton $\mathrm{X}-100$ for $5 \mathrm{~min}$ at $25^{\circ} \mathrm{C}$. GDH activity was measured after addition of $10 \mathrm{mM} \alpha$-ketoglutarate and was monitored by the oxidation of $\mathrm{NADH}$, ie, reduction in UVabsorption at $340 \mathrm{~nm}$ at $25^{\circ} \mathrm{C}$ using a spectrophotometer DU-60 (Beckman, Fullerton, CA, USA). ${ }^{14,15}$ Reaction mixture was $100 \mu \mathrm{g}$ cell lysate. GTP and ADP were added to the reaction mixtures for the allosteric effect of GDH. Protein concentration was measured by using the Dc Protein Assay (Bio-Rad, Richmond, CA, USA). 


\section{Identification of human GDH mutations}

Genomic DNA was isolated from lymphoblasts transformed with EBV. Each exon and its flanking intronic region were amplified with a pair of human GDH-specific oligonucleotide primers (one primer was biotinylated) using polymerase chain reaction (PCR), as previously described. ${ }^{16}$ The amplified products were purified to single-strand DNA using magnetic beads coated with streptavidin M280 (Dynal, Oslo, Norway). The purified single-stranded DNA was sequenced by the dye terminator method using an ABI PRISM 310 Genetic Analyzer (Perkin Elmer, Norwalk, CT, USA). Primers used for the PCR amplification in exon 10 and 12 of the human GDH gene (GenBank accession numbers X66300-66312) ${ }^{17,18}$ were as follows: for exon 10, biotin-sense primer of $5^{\prime}$-GTGTGAAATTCCTGATACTGGCT$3^{\prime}$ and antisense primer of 5'-GTGAGTTTGGCGAACAAGATTAT-3'. Sequencing primer was sense primer of 5'-AGGTTCATTTTCAGCTTTTAC-3'. For exon 12, biotin-antisense primer of 5'-ACAGTCTGGCGGCTGAGATAG-3' and sense primer of 5'-ACAGGGACACAAAGCAGGTC-3'. Sequencing primer was antisense primer of $5^{\prime}$-ATGGTTGAGTTGCACTTCAT-3'.

\section{Expression analysis}

Full-length human glutamate dehydrogenase cDNA from human hepatocytes was kindly provided by Dr Amuro, Nippon Medical School. ${ }^{17}$ Mutant human GDH cDNA was synthesized by specific base substitutions using site-directed mutagenesis into a eukaryotic expression vector (pCDNA3; Invitrogen, Carlsbad, CA, USA) containing a full-length human GDH cDNA. Mutant or normal GDH cDNAs were introduced into COS cells by electroporation with a Gene Pulser (Bio-Rad) at $200 \mathrm{~V}$ with $960 \mu \mathrm{F}$ capacitance, as described previously. ${ }^{16}$ The cells were harvested after $72 \mathrm{~h}$ culture. GDH activity in the homogenates of COS cells transfected with mutant and normal constructs was determined in duplicate as described above to ensure reproducibility. The mitochondrial fraction of COS cells was used for Western blot analysis. The extracts were electrophoresed in SDS-polyacrylamide gel and subsequently transferred to GeneScreen hybridisation transfer membrane (Du-PointNEN, Boston, MA, USA). Immunoreactive GDH was identified by using primary antibody of a rabbit anti-calf GDH antibody (Amersham Pharmacia Biotech, Uppsala, Sweden), secondary antibody of a goat anti-rabbit IgG-peroxidase (Roche Diagnostics, Mannheim, Germany), and ECL system (Amersham Pharmacia Biotech). The density of each band was determined by scanning with a Bio-Rad Imaging Densitometer (GS-700). GDH mRNA in cell extracts was determined by dot-blot hybridisation for serially diluted total-RNA samples with a GDH cDNA probe labelled with $\alpha-{ }^{32}$ P-dCTP (Du-Point-NEN) using the Megaprime DNA labelling system (Amersham Pharmacia Biotech). GDH activity and immunoreactive protein level were normalised with transfection efficiency by GDH mRNA levels.

\section{Results}

\section{GDH activity}

The activity and allosteric response of GDH in lymphoblasts from the patients and control subjects are shown in Figure 1. The basal GDH activity of patient $1(33.4 \mathrm{nmol} / \mathrm{mg}$ of protein/min) was twofold higher than those of the controls (16.9). Patient 2 (20.2) was the same as the controls. The inhibitory effects of GTP in patients 1 and 2 were less than the control, and the half-maximal inhibitory concentrations of GTP in the control subjects, patient 1 , and patient 2 were $0.17,0.24$, and $0.87 \mu \mathrm{mol} / 1$, respectively (Figure $1 \mathrm{~A}$ ). The insensitivity for GTP inhibition was mild in patient 1 while severe in patient 2 . In the sensitivity for ADP stimulation, GDH activity of patient 1 was twofold higher at basal level and was within normal upper limit at maximal stimulation. Patient 2 was similar to the control subjects (Figure 1B).

\section{Identification of GDH mutation}

To characterise GDH genes in the two patients, we performed sequence analysis in the two families (Figure 2). The mutation in patient 1 was heterozygous T-to-G transition at nucleotide position 1396 in exon 10, resulting in the replacement of leucine (TTG) by valine (GTG) at codon 413 (L413V). Patient 2 showed heterozygous G-to-C transition at nucleotide position 1509 in exon 12, resulting in the replacement of glycine (GGT) by asparagine (GAT) at codon 446 (G446D). In both parents, we could not find any substitutions as seen in each patient. The above results indicate that both mutations occurred de novo and were observed only in a single allele. L413V in exon 10 was a novel mutation, while G446D in exon 12 was identified in Caucasians by Stanley et al. ${ }^{9}$

Confirmation of mutation by expression analysis

To determine whether the L413V and G446D substitutions were associated with GDH activities in lymphoblasts and to assess the potential GDH activities of both substitutions, we
A

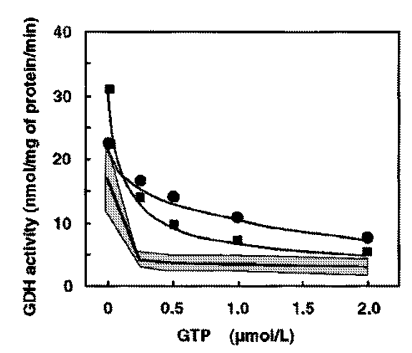

B

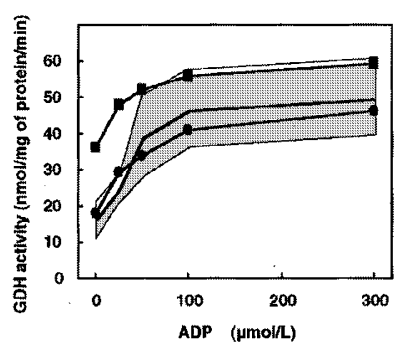

Figure 1 Effects of GTP and ADP on GDH activity in lymphoblasts of control subjects, patient 1 and 2. (A) Effects of GTP. (B) Effects of ADP. Closed squares: activity of GDH in patient 1 , closed circles: activity of GDH in patient 2 . The shaded area represents the mean $\pm S D$, and centre line the mean of six control subjects. 

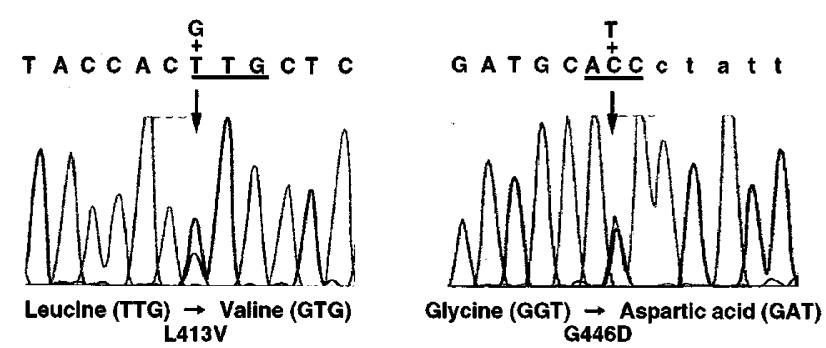

Figure 2 Identification of two missense mutations in two patients with $\mathrm{CHH}$. (A) Two bands ( $\mathrm{T}$ and $\mathrm{G}$ ) are present at the same position with the sequence of sense direction in patient 1. This $T$ to $G$ transition at nucleotide 1396 of the GDH cDNA in exon 10 results in the replacement of leucine (TTG) by valine (GTG). (B) Two bands (C and T) are present at the same position with the sequence of antisense direction in patient 2 . This $C(G)$ to $\mathrm{T}(\mathrm{A})$ transition at nucleotide 1509 in exon 12 results in the replacement of glycine (GGT) by aspartic acid (GAT).

reconstructed each mutant in the expression vector pCDNA3 by site-directed mutagenesis. The GDH activities and immunoreactive amounts of the normal and mutant constructs were established by correcting the transfection efficiency into COS cells. The allosteric response of GDH mutant and normal constructs are shown in Figure 3 . The normal GDH cDNA in COS cells with a transient expression system led to 10-fold stimulation of $\mathrm{GDH}$ activity $(117.0 \mathrm{nmol} / \mathrm{mg}$ of protein/min) compared to the endogenous background (11.5). The basal GDH activities in COS cells transfected with the L413V constructs (217.2) were twofold higher than those of normal and G446D constructs (117.0 and 98.1, respectively), which were similar to those in lymphoblasts. The half-maximal inhibitory concentrations of GTP in L413V and G446D constructs were 1.23 and $1.97 \mu \mathrm{mol} / \mathrm{l}$, respectively, which were $2-5$-fold higher than those in lymphoblasts of patients. On the other hand, the GTP concentrations of mock and normal constructs were 0.21 and $0.18 \mu \mathrm{mol} / 1$, which were similar to those in lymphoblasts. The immunoreactive proteins of both L413V and G446D constructs appeared as a single band and showed the same position and amount as those of normal constructs. Thus, these results define the L413V and G446D substitutions as the causal genes for impairment of GDH activity in the two patients, and indicated that the abnormal allosteric responses of GDH did not correlate with the amount of GDH protein.

\section{Discussion}

In the present study, we identified deterioration of GDH regulation in lymphoblasts of two patients with hyperinsulinism and hyperammonaemia. We also identified missense mutations of L413V in patient 1 and G446D in patient 2. These findings are consistent with the aetiology of this disease; the altered form of GDH leads to excessive insulin

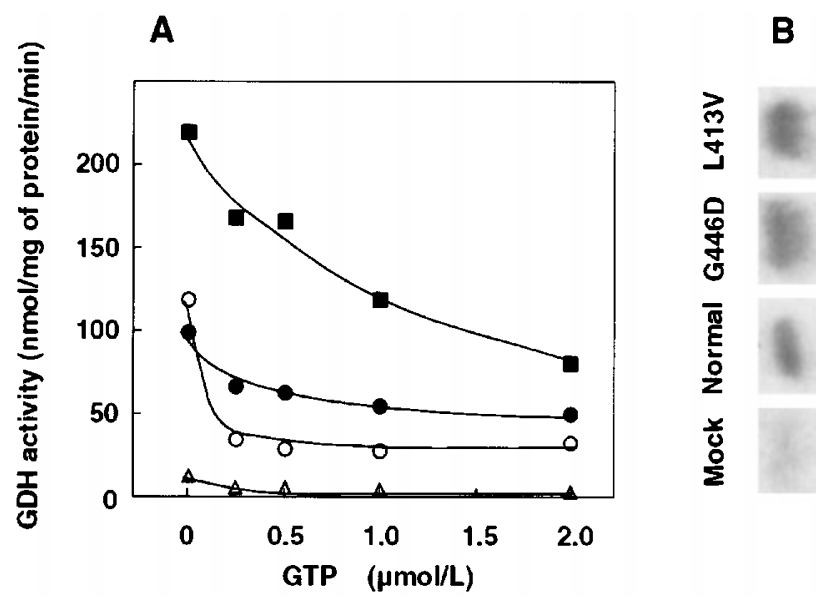

Figure 3 Analysis of immunoreactive protein and GDH activity in COS cells transfected with normal or mutant cDNAs constructs by expression analysis. (A) GDH activity from L413V (closed squares) and G446D (closed circles), normal constructs (open circles), and mock (open triangles) were shown.

(B) Immunoreactive GDH proteins from normal, L413V, and G446D constructs were identified by Western blotting.

release from pancreatic $\beta$-cells and insufficient processing of ammonia in the liver. ${ }^{9}$ In our experiments, patient 2 with G446D mutation showed severe insensitivity for GTP inhibition. The basal GDH activity and allosteric effect of ADP stimulation in patient 2 was the same as the control subjects, as described previously. ${ }^{9,19,20}$ However, in patient 1 , the dysregulation of GDH caused by L413V mutation was different from G446D mutation inside the GTP-binding site of exons 11 and 12. This dysregulation in patient 1 was due to twofold higher basal activity and mild insensitivity for GTP inhibition in GDH. Similar to our findings, Yorifuji et $a l^{21}$ found a patient with severe CHH phenotype. The GDH activity in lymphoblasts with heterozygous N410T mutation appears to be approximately twofold higher than controls and maintained its responses to GTP inhibition and ADP stimulation. It is suspected that the dysregulation of L413V and N410T mutations outside the GTP-binding site of exons 11 and 12 are associated with different mechanism and different conformational effects from previous mutations inside the GTP binding site. The mechanism of allosteric control in mutations could be solved by the X-ray crystal structure of bovine GDH. Peterson et $a l^{22}$ and Smith et $a l^{23}$ proposed that the catalytic cleft between NAD-binding domain and glutamate-binding domain is narrowed by GTP and is widened by ADP on a hinge of the pivot helix (Figure 4). They also found the unique structure of 'antenna' connecting to the pivot helix in bovine GDH. The mutations found from patients with $\mathrm{CHH}$ can be divided into three sites according to the location on the structure of GDH. Site 1 is in 15 amino acids between F440 and H454 in human within exons 11 and 12. On the structure of GDH, this site is on the pivot helix and $\mathrm{H} 450$ on bovine GDH 


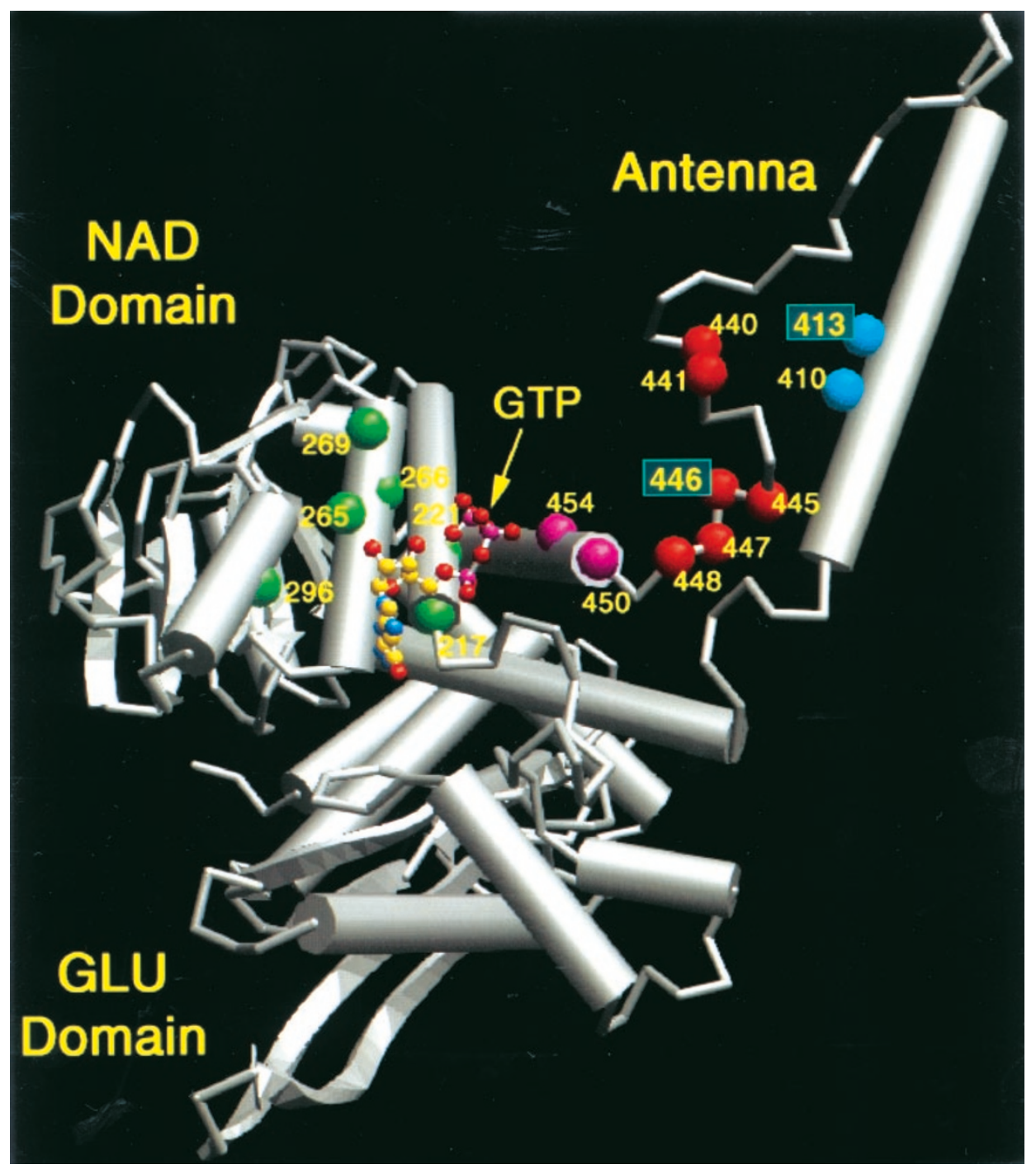

Figure 4 The location of L413V, and G446D mutations in $\mathrm{CHH}$. One subunit of the GDH homohexamer represented as a ribbon diagram on the basis of the X-ray crystallographic structure of bovine $\mathrm{GDH}^{22,23}$ The catalytic cleft is between NAD domain and Glutamate domain. GTP molecule is represented by a ball-and-stick model. Mutated amino acid residues are shown as purple balls in the pivot helix, red balls in GTP binding site of exons 11 and 12, green balls in GTP binding site of exons 6 and 7, and blue balls in front site of antenna. Our mutations of L413V and G446D are shown in green box. The figure was kindly prepared by Thomas J Smith, using MolView. ${ }^{26}$

making contact with the triphosphate of GTP and its surroundings. All mutations from the patients with $\mathrm{CHH}$ were found in this site 1 in the first and second reports by Stanley et $a l,{ }^{9,19}$ which predicted the region of the binding peptides to GTP by the photoaffinity labelling method. ${ }^{11}$ Site 2 is in exons 6 and 7, which interacts with GTP molecules. On the structure of GDH, the triphosphate of GTP makes contact with H209, R217, R261, Y262, R265, and $\mathrm{H} 450$ on bovine GDH and the purine ring of GTP interacts with E292 and K289 on bovine GDH (bovine GDH is 4 amino acids shorter in N-terminal than human GDH). $\mathrm{E} 296 \mathrm{~A}$ and $\mathrm{R} 265 \mathrm{~L},{ }^{24} \mathrm{R} 221 \mathrm{C}$ and $\mathrm{R} 269 \mathrm{H},{ }^{20}$ and $\mathrm{S} 217 \mathrm{~L}$,
R256T, Y266C, and R269C ${ }^{25}$ in exons 6 and 7 have been identified outside the above GTP binding site 1 . The mechanism of hyperinsulinism and hyperammonaemia caused by mutations in sites 1 and 2 is clearly explained by the insensitivity for the GTP inhibition on GDH enzyme. Site 3 is in exon 10, which is located in the $\alpha$-helix of the antenna-like structure on the other side from the hinge. Only two mutations of L413V and N410T have been found in this site 3 . The similarity of GDH regulation in both mutations is that both have about twofold higher basal GDH activity than control subjects. The difference between L413V and N410T is the insensitivity for GTP inhibition and the 
reaction for ADP stimulation. These findings suggest that the antenna-like structure plays an important role in GDH activity.

In COS cell expression analysis, L413V and G446D mutant constructs showed insensitivity to the inhibitory effect of GTP. Therefore, they were confirmed to be the causative mutations for $\mathrm{CHH}$. Furthermore, the expression analysis allowed the demonstration of the dosage effect of mutant GDH gene, indicating a gain-of-function. The half-maximal inhibitory concentrations of GTP for both mutant constructs in COS cells were 2-5-fold higher than that in lymphoblasts from the patients. Electroporation of COS cells with normal GDH cDNA in the transient expression assay resulted in 10fold greater stimulation of GDH activity over the endogenous background. Therefore, COS cells with the mutant constructs were similar to the homozygous state for each mutation, while lymphoblasts obtained from the patients were heterozygous for mutant/normal. The level of insensitivity of allosteric inhibition by GTP was proportionate to the increase in the amounts of mutant genes. In addition, the amounts of immunoreactive proteins for L413V and G446D mutations were similar to those of normal construct, suggesting that increased basal GDH activity and changes in inhibitory effects in mutant GDH depend not on the amount of enzyme but on the difference in specific activity.

The potency of the allosteric inhibition of GTP in lymphoblasts of patient 1 (L413V) and in COS cells transfected with L413V constructs was between that of control subjects and patient 2 (G446D), even though basal activities in patient 1 (L413V) were twofold higher than the controls, ie, impairment of GDH regulation in those with L413V was obviously less severe than in those with G446D. The hypoglycaemic symptoms in patient 1 with L413V appeared at 4 months of age, definitely later than that in patient 2 with G446D and hypoglycaemia was relatively easily controllable in patient 1 . In addition, the serum ammonium levels in patient 1 were significantly lower $(P<0.0001)$ than those in patient 2 in the last 3 years. The clinical phenotype was mild in patient 1 . These findings indicate a close correlation among the severity of clinical symptoms (clinical phenotype), the level of impaired GDH regulation in lymphoblasts (biochemical phenotype), and the level of impaired regulation of GDH expression (genotype). On the other hand, clinical phenotypes in some reported cases with $\mathrm{CHH}$ were not consistent with the biochemical phenotype determined by GDH enzymatic analysis and genotype. For example, two patients with G446D mutation were identified by familial analysis who were otherwise asymptomatic. ${ }^{9}$ Similarly, other reported cases with A447T developed hypoglycaemic seizures and died in early infancy, whereas others with a similar abnormality survived into adulthood without ever showing signs of hypoglycaemia. ${ }^{19}$ Thus, it is possible that other genetic or environmental factors are involved in the development of hyperinsulinism and hyperammonaemia.
Further studies are necessary to investigate prospectively the relationship between clinical symptoms and genotype and other genetic and environmental factors involved in the development of $\mathrm{CHH}$.

\section{Acknowledgments}

We thank Dr Thomas J Smith, Department of Biological Sciences, Purdue University, for generously providing information on the X-ray crystal structure of bovine GDH. This study was supported in part by a grant from the Ministry of Health and Welfare of Japan, and in part by a fund from the Mother and Child Health Foundation and Suyama Research Foundation.

\section{References}

1 Glaser B, Kesavan P, Heyman M et al: Familial hyperinsulinism caused by an activating glucokinase mutation. $N$ Engl J Med 1998; 338: $226-230$.

2 Thomas PM, Cote GJ, Wohllk $\mathrm{N}$ et al: Mutations in the sulfonylurea receptor gene in familial persistent hyperinsulinemic hypoglycemia of infancy. Science 1995; 268: 426-429.

3 Dunne MJ, Kane C, Shepherd RM et al: Familial persistent hyperinsulinemic hypoglycemia of infancy and mutations in the sulfonylurea receptor. N Engl J Med 1997; 336: 703 - 706.

4 Nestorowicz A, Wilson BA, Schoor KP et al: Mutations in the sulfonylurea receptor gene are associated with familial hyperinsulinism in Ashkenazi Jews. Hum Mol Genet 1996; 5: $1813-$ 1822.

5 Thomas P, Ye YY, Lightner E: Mutations of the pancreatic islet inward rectifier Kir6.2 also leads to familial persistent hyperinsulinemic hypoglycemia of infancy. Hum Mol Genet 1996; 5: $1809-1812$.

6 Zammarchi E, Filippi L, Novembre E, Donati MA: Biochemical evaluation of a patient with a familial form of leucine-sensitive hypoglycemia and concomitant hyperammonemia. Metabolism 1996; 45: 957 - 960 .

7 Weinzimer SA, Stanley CA, Berry GT, Yudkoff M, Tuchman M, Thornton PS: A syndrome of congenital hyperinsulinism and hyperammonemia. J Pediatr 1997; 130: 661-664.

8 Kitaura J, Miki Y, Kato H, Sakakihra Y, Yanagisawa M: Hyperinsulinaemic hypoglycaemia associated with persistent hyperammonaemia. Eur J Pediatr 1999; 158: 410-413.

9 Stanley CA, Lieu YK, Hsu BY et al: Hyperinsulinism and hyperammonemia in infants with regulatory mutations of the glutamate dehydrogenase gene. N Engl J Med 1998; 338: 13521357.

10 Shoemaker MT, Haley BE: Identification of a guanine binding domain peptide of the GTP binding site of glutamate dehydrogenase: isolation with met al:-chelate affinity chromatography. Biochemistry 1993; 32: $1883-1890$.

11 Cho SW, Ahn JY, Lee J, Choi SY: Identification of a peptide of the guanosine triphosphate binding site within brain glutamate dehydrogenase isoproteins using 8-azidoguanosine triphosphate. Biochemistry 1996; 35: $13907-13913$.

12 Stanley CA: Hyperinsulinism in infants and children. Pediatr Clin N Am 1997; 44: 363 -374.

13 Brusilow SW, Horwich AL: Urea cycle enzymes. In: Scriver C, Beaudet A, Sly W, Valle D (eds): The Metabolic and Molecular Bases of Inherited Disease. 8th edn. New York: McGraw-Hill, 2000, pp $1909-1963$.

14 Bryta J, Michalik M, Nelson J, Erencinska M: Regulation of the glutamate dehydrogenase activity in rat islets of Langerhans and its consequence on insulin release. Metabolism 1994; 43: $1187-1195$ 
15 Wrzeszczynski KO, Colman RF: Activation of bovine liver glutamate dehydrogenase by covalent reaction of adenosine 5'-O-[S-(4-bromo-2,3-dioxobutyl) thiophosphate] with arginine-459 at an ADP regulatory site. Biochemistry 1994; 33: $11544-11553$.

16 Asada M, Okano Y, Imamura T, Suyama I, Hase Y, Isshiki G: Molecular characterization of galactokinase deficiency in Japanese. J Hum Genet 1999; 44: 377-382.

17 Amuro N, Goto Y, Okazaki T: Isolation and characterization of the two distinct genes for human glutamate dehydrogenase. Biochim Biophys Acta 1990; 1049: 216-218.

18 Michaelidis TM, Tzimagiorgis G, Moschonas NK, Papamatheakis J: The human glutamate dehydrogenase gene family: gene organization and structural characterization. Genomics 1993; 16: $150-160$.

19 Stanley CA, Fang J, Kutyna K et al: Molecular basis and characterization of the hyperinsulinism/hyperammonemia syndrome: predominance of mutations in exons 11 and 12 of the glutamate dehydrogenase gene. Diabetes 2000; 49: $667-$ 673.

20 Santer R, Kinner M, Passarge M et al: Novel missense mutations outside the allosteric domain of glutamate dehydrogenase are prevalent in European patients with the congenital hyperinsulinism-hyperammonemia syndrome. Hum Genet 2001; 108: 66 71.
21 Yorifuji T, Muroi J, Uematsu A, Hiramatsu H, Momoe T: Hyperinsulinism-hyperammonemia syndrome caused by mutant glutamate dehydrogenase accompanied by novel enzyme kinetics. Hum Genet 1999; 104: 476-479.

22 Peterson P, Smith TJ: The structure of bovine glutamate dehydrogenase provides insight into the mechanism of allostery. Structure 1999; 7: 769-782.

23 Smith TJ, Peterson PE, Schmidt T, Fang J, Stanley CA: Structures of bovine glutamate dehydrogenase complexes elucidate the mechanism of purine regulation. J Mol Biol 2001; 307: 707 - 720.

24 Miki Y, Taki T, Ohura T, Kato H, Yanagisawa M, Hayashi Y: Novel missense mutations in the glutamate dehydrogenase gene in the congenital hyperinsulinism-hyperammonemia syndrome. $J$ Pediatr 2000; 136: 69-72.

25 MacMullen C, Fang J, Hsu BY et al: Hyperinsulinism/hyperammonemia syndrome in children with regulatory mutations in the inhibitory guanosine triphosphate-binding domain of glutamate dehydrogenase. J Clin Endocrinol Metab 2001; 86: $1782-1787$.

26 Smith TJ: MolView: a program to analyze and display atomic structures on the Macintosh personal computer. J Mol Graph 1995; 13: 122-125. 